\title{
Mapping the magic numbers in binary Lennard-Jones clusters
}

\author{
Jonathan P. K. Doye and Lars Meyer \\ University Chemical Laboratory, Lensfield Road, Cambridge CB2 1EW, United Kingdom
}

(Dated: October 9, 2018)

\begin{abstract}
Using a global optimization approach that directly searches for the composition of greatest stability, we have been able to find the particularly stable structures for binary Lennard-Jones clusters with up to 100 atoms for a range of Lennard-Jones parameters. In particular, we have shown that just having atoms of different size leads to a remarkable stabilization of polytetrahedral structures, including both polyicosahedral clusters and at larger sizes structures with disclination lines.
\end{abstract}

PACS numbers: $61.46 .+\mathrm{w}, 36.40 . \mathrm{Mr}$

The structure of binary clusters has been the subject of much recent interest, both because of the technological importance of alloy clusters, such as in catalysis, and the opportunity to tailor the structure through the choice of atom types and composition [1], potentially leading to novel structural forms, such as the core-shell structures recently found for silver alloy clusters [2]. Binary clusters also offer considerable additional challenges to the theoretician, compared to the one-component case. Firstly, for a given cluster, there are many more minima on the potential energy surface, because of the presence of "homotops" [3], isomers with the same geometric structure, but which differ in the labelling of the atoms. Secondly, the composition provides an additional variable that adds to the complexity of the structural behaviour.

For example, the task of obtaining the lowest-energy structures for all compositions and all sizes up to 100 atoms would require 5050 different global minima to be found. Our approach here is different, as normally one is not interested in all these possible structures, but only the most stable. Therefore, in our global optimization runs the composition is allowed to change, and so we attempt to find the cluster at a given size with the optimal composition directly. Thus, the task has been reduced back down to finding one global minimum for each size, as for the one-component case. Of course, the search space for each optimization is extremely large, and so it is very important to make extensive use of moves that change the identity of atoms [4] in order to search the space of homotops and different compositions as efficiently as possible.

The focus of the current work is on how different structures, particularly those that are polytetrahedral 5], can be stabilized just through the two atom types in the cluster having different sizes [2]. In polytetrahedral structures all the occupied space can be divided up into tetrahedra with atoms at their corners. However, regular tetrahedra cannot pack all space, and so polytetrahedral packings are said to be frustrated. For example, in the 13-atom icosahedron, which can be considered to be made up of 20 tetrahedra sharing a common vertex, the distance between adjacent atoms on the surface is $5.15 \%$ longer than that between the central atom and a

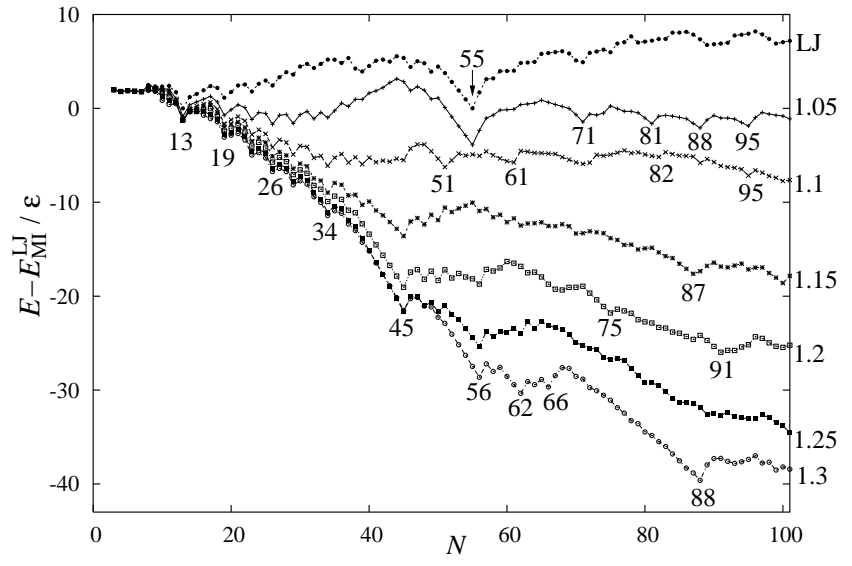

FIG. 1: The energy of the BLJ global minimum for the six values of $\sigma_{B B} / \sigma_{A A}$ studied, relative to $E_{\mathrm{MI}}^{\mathrm{LJ}}$, a fit to the energies of the Mackay icosahedra for LJ clusters. A line corresponding to the LJ global minima is also included

surface atom. However, the associated strain can be removed by choosing the central atoms to be $9.79 \%$ smaller [6]. Similarly, Frank-Kasper phases, bulk polytetrahedral crystals, are only found for alloys [7].

To achieve our aims we use a binary Lennard-Jones (BLJ) potential:

$$
E=4 \sum_{i<j}\left(\frac{\sigma_{\alpha \beta}}{r_{i j}}\right)^{12}-\left(\frac{\sigma_{\alpha \beta}}{r_{i j}}\right)^{6},
$$

where $\alpha$ and $\beta$ are the atom types of atoms $i$ and $j$, respectively. To study the effects of different atom sizes, independent of energetic effects, we choose $\epsilon_{A A}=$ $\epsilon_{A B}=\epsilon_{B B}=\epsilon$ and define $\sigma_{A B}$ using the Lorentz rule: $\sigma_{A B}=\left(\sigma_{A A}+\sigma_{B B}\right) / 2$. The one parameter in the potential is then $\sigma_{B B} / \sigma_{A A}$. For this choice of parameters a tendency to form core-shell clusters has been observed [8, 9], but no systematic structural survey has been made. Our aim here is to find how the stable structures of the BLJ clusters change, as $\sigma_{B B} / \sigma_{A A}$ varies in the range 1.0 to 1.3 for all clusters with up to 100 atoms.

The energies of the putative global minima that we have found are depicted in Figures 1 and 2] where Fig. 1 


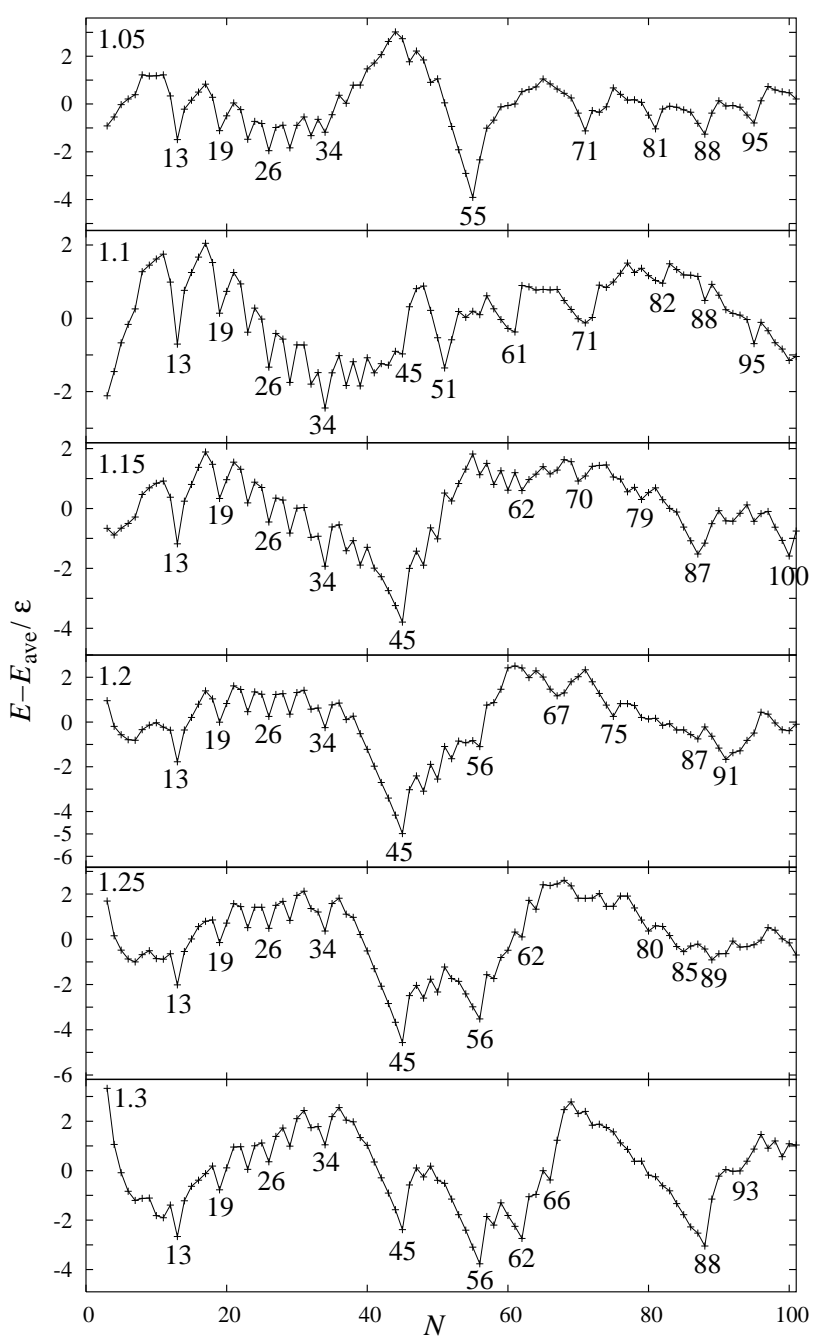

FIG. 2: The six panels correspond to the energy of the global minimum for the six values of $\sigma_{B B} / \sigma_{A A}$ studied, relative to $E_{\text {ave }}$, a fit to the energies of the global minima at that size ratio.

compares the energies at different values of $\sigma_{B B} / \sigma_{A A}$ and Fig. 2] allows the magic numbers at each size ratio to be identified more easily. Figure 3 provides a more detailed analysis of the behaviour of $\mathrm{BLJ}_{13}$, Figure 4 shows a selection of particularly stable structures and Figure 5 how the structural form of the global minima depends on $N$ and $\sigma_{B B} / \sigma_{A A}$. The energies and points files for all the global minima are available online [10].

The reference system to which our results are compared is the one-component Lennard-Jones (LJ) clusters, for which the structural behaviour is well-understood. In the present size range, the LJ global minima are dominated by structures based upon the Mackay icosahedra 11. These Mackay icosahedra are made up of twenty face-centred-cubic (fcc) tetrahedra sharing a common vertex, and except for the smallest icosahedron at $N=13$ are not polytetrahedral.

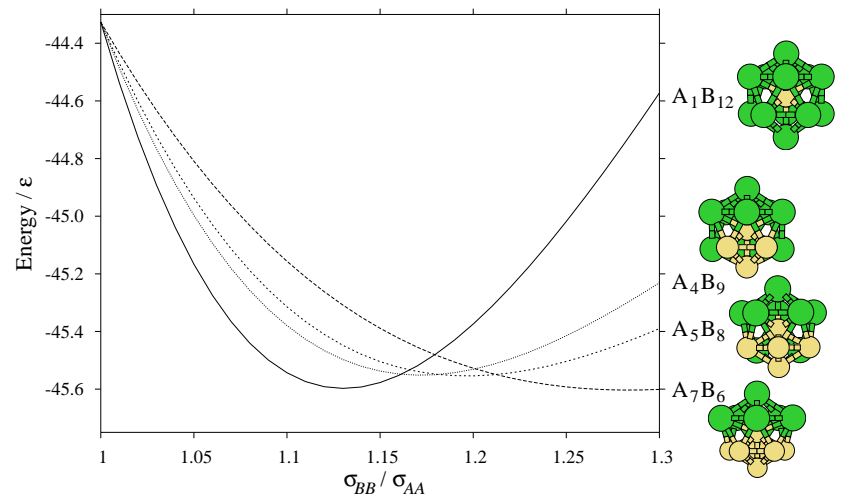

FIG. 3: (Colour online) The dependence of the energies of the four BLJ $\mathrm{BJ}_{13}$ global minima on $\sigma_{B B} / \sigma_{A A}$.

Growth upon the Mackay icosahedra can occur in two ways. The first, the anti-Mackay overlayer, consists in adding atoms in sites that are hexagonal close-packed with respect to the underlying fcc tetrahedra and above the twelve vertices. For the 13-atom icosahedron, this overlayer maintains the polytetrahedral character of the clusters. The second, the Mackay overlayer continues the fcc packing of the underlying tetrahedra and leads to the next Mackay icosahedron. Growth initially occurs in the anti-Mackay overlayer because of a greater number of nearest-neighbour contacts, but before this overlayer is complete, the LJ global minimum changes to Mackay character [11], because of the greater strain energy associated with the anti-Mackay overlayer.

It is immediately clear from Fig. 1 that allowing atoms of different sizes leads to a dramatic stabilization of the clusters. For example, the $\mathrm{BLJ}_{45}$ global minimum at $\sigma_{B B} / \sigma_{A A}=1.3$ is $26.9 \epsilon$ or $12.6 \%$ lower in energy than that for $\mathrm{LJ}_{45}$. The origins of this stabilization are quickly apparent from an analysis of the structural behaviour of the BLJ clusters. The structural phase diagram in Fig. [5 shows that for the majority of the parameter space, the global minima are polytetrahedral. Only in the bottom right-hand corner (large $N$ and low size ratio) are nonpolytetrahedral structures most stable. For LJ clusters, such polytetrahedral structures are disfavoured beyond 30 atoms because of their greater strain energy, however the presence of different-sized atoms relieves this strain. As the polytetrahedral structures generally have a greater number of nearest neighbours, they therefore become lowest in energy.

Perhaps surprisingly, there is no optimal size ratio, but instead the energy virtually monotonically decreases with increasing $\sigma_{B B} / \sigma_{A A}$ (Fig. (1). For example, based on the analysis of the geometry of the icosahedron mentioned earlier, one might expect a size difference near $10 \%$ to be optimal for $\mathrm{BLJ}_{13}$. Indeed, the energy of $\mathrm{A}_{1} \mathrm{~B}_{12}$ shows a pronounced minimum near this value (Fig. B3), however just beyond this minimum the optimal structure 


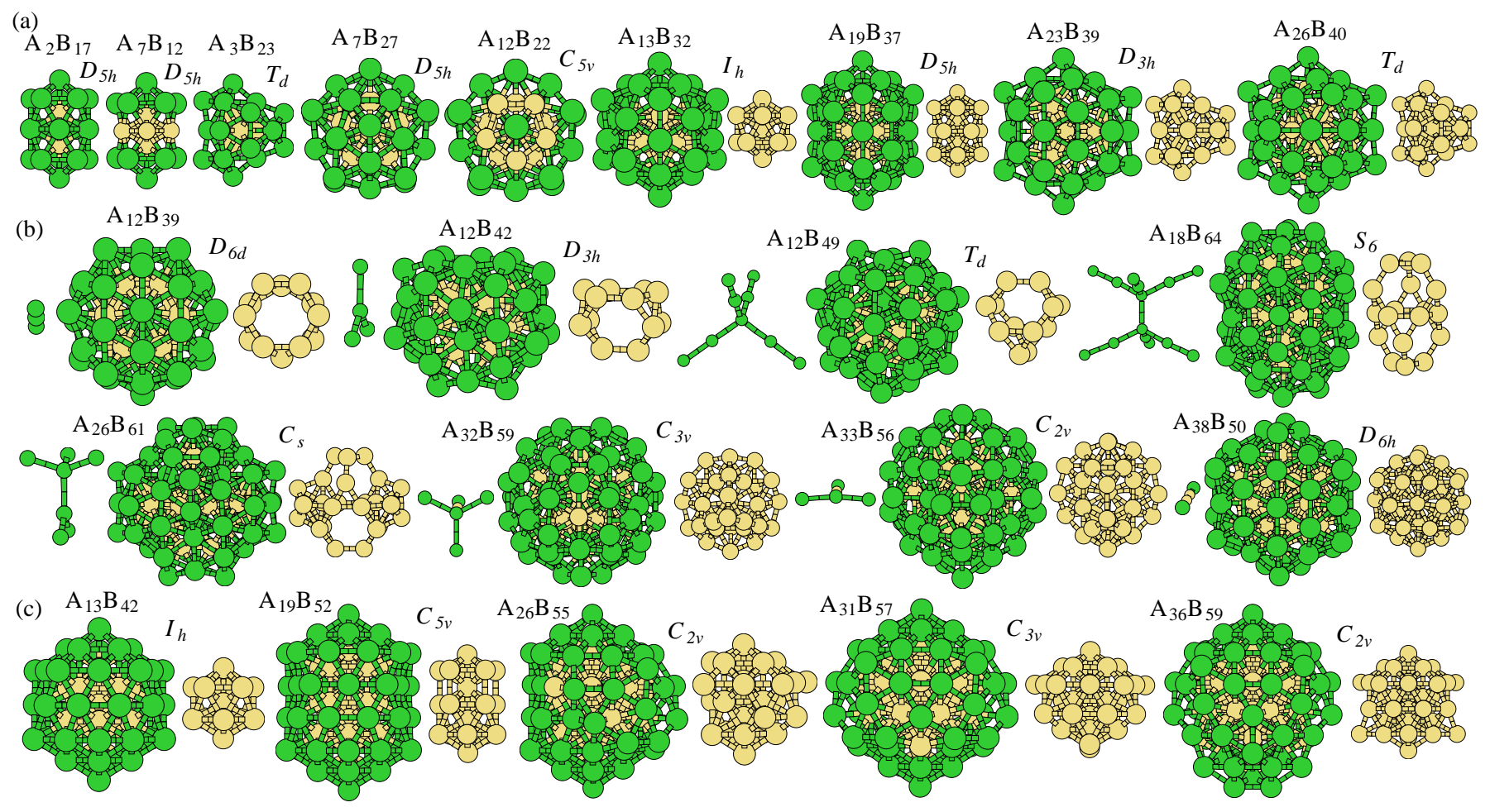

FIG. 4: (Colour online) A selection of the particularly stable BLJ global minima grouped according to their structural type: (a) polyicosahedral, (b) polytetrahedral with disclinations and (c) the 55-atom Mackay icosahedron with an anti-Mackay overlayer. For the larger clusters, to the right of the cluster, the A-atom core is also depicted, and in (b) to the left is the disclination network.

changes. The low energy is maintained by adding an increasing number of the smaller A atoms into the surface of the icosahedron, as this keeps the nearest-neighbour distances near to their optimal values. A similar story holds for larger clusters, except that the optimal values of $\sigma_{B B} / \sigma_{A A}$ for the core-shell geometry are larger (Fig. (5) because greater size ratios are needed to fully relieve the strain.

This preference for polytetrahedral structures is evident in the magic numbers (Fig. 21). Only for $\sigma_{B B} / \sigma_{A A}=1.05$ is the 55-atom Mackay icosahedron still a magic number. Instead the magic numbers at $N=19$, $23,26,29,34$, and 45 associated with the covering of the 13-atom icosahedron by the anti-Mackay overlayer become increasingly prominent. These structures are polyicosahedral - each atom in the interior of the cluster has a local icosahedral coordination shell - and are made up of 2, 3, 4, 5, 7 and 13 interpenetrating icosahedra, respectively (Fig. 4). Recently, similar polyicosahedral core-shell structures have been found for alloy clusters of silver [2]. Although a significant proportion of the polyicosahedral region of the structural phase diagram corresponds to core-shell clusters (Fig. 5), the stability of these structures is not dependent on such an arrangement, and as for $\mathrm{BLJ}_{13}, \mathrm{~A}$ atoms are incorporated into the surface at larger $\sigma_{B B} / \sigma_{A A}$, as illustrated by $\mathrm{A}_{7} \mathrm{~B}_{12}$ and $\mathrm{A}_{12} \mathrm{~B}_{22}$ in Fig. 目

The complete anti-Mackay covering of the 13-atom icosahedron occurs at $N=45$, however at larger size ratios the polyicosahedral growth continues beyonds this size. The magic numbers at $N=56,62$ and 66 correspond to core-shell polyicosahedral structures with the double, triple and quadruple icosahedra mentioned above as their core (Fig. 4).

As $N$ increases the polyicosahedral structures have increasingly large tensile strains in the surface, hence the need for increasingly large size ratios to counteract this. Furthermore, polyicosahedral structures are impossible for bulk. Instead, the Frank-Kasper phases also involve coordination numbers greater than 12 . Such structures can be described using disclinations, where a disclination runs along those edges in the structure that have six tetrahedra surrounding them, rather than the usual five for icosahedral coordination [5]. Polytetrahedral clusters involving disclinations introduce both tensions and compressions into the structure, and represent a better compromise at larger $N$. Indeed, such structures cover a significant proportion of the phase diagram at larger $N$ (Fig. [5), and some examples are illustrated in Fig. 团

Unlike the polyicosahedral structures, the core of the cluster is not necessarily made up completely of A atoms, but instead the atoms with coordination number $(Z)$ 


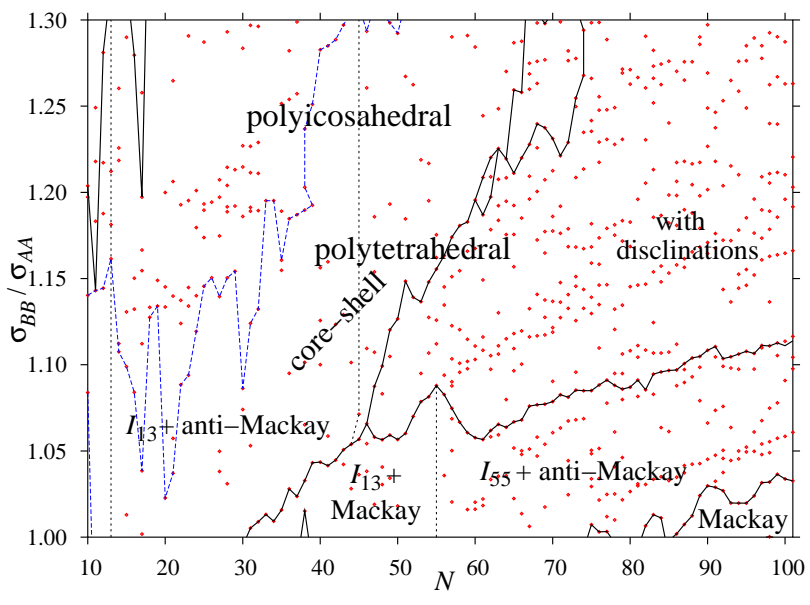

FIG. 5: (Colour online) Structural phase diagram showing how the structure of the global minimum depends on $N$ and $\sigma_{B B} / \sigma_{A A}$. Each point corresponds to the value of $\sigma_{B B} / \sigma_{A A}$ at which the global minimum for a given $N$ changes. The lines divide the diagram into regions where the global minima have the same structural type. The labels $I_{13}$ and $I_{55}$ stand for the 13- and 55-atom Mackay icosahedra.

greater than 12 usually correspond to the larger B atoms. Atoms with $Z=14$ have a single disclination running through them, whilst atoms with $Z=15$ and 16 are nodes for three and four disclinations, respectively.

Some of the polytetrahedral magic numbers at $\sigma_{B B} / \sigma_{A A}=1.1$ have been previously seen for onecomponent clusters interacting with long-ranged Morse [12] and modified Dzugutov 13] potentials. The structures at $N=51,54$ and 61 consist of the $Z=14,15$ and 16 coordination polyhedron, respectively, covered by a (near-)complete anti-Mackay-like overlayer. As such, they are the high coordination number analogues of $\mathrm{A}_{13} \mathrm{~B}_{32}$. Similarly, $\mathrm{A}_{18} \mathrm{~B}_{64}$ is the analogue of $\mathrm{A}_{19} \mathrm{~B}_{37}$, but with two interpenetrating $\mathrm{Z}=16$ coordination polyhedra at the centre.

As $\sigma_{B B} / \sigma_{A A}$ increases, the fraction of the atoms that lie on disclination lines decreases, reaching zero at the polyicosahedral boundary in the structural phase diagram. This trend is illustrated by the four structures in the second line of Fig. 目(b), which correspond to magic numbers for $\sigma_{B B} / \sigma_{A A}=1.15,1.2,1.25$ and 1.3 , respectively. For example, the hexagonal disk structure that occurs for $\sigma_{B B} / \sigma_{A A}=1.3$ at $N=88$, has a single disclination running along the six-fold symmetry axis, and its A-atom core is a 38-atom structure previously seen for Dzugutov clusters 13, 14].

The major non-polytetrahedral portion of the structural phase diagram corresponds to structures based on the 55-atom Mackay icosahedron. As for growth on the 13-atom icosahedron, the size at which the transition from an anti-Mackay to a Mackay overlayer occurs increases with increasing $\sigma_{B B} / \sigma_{A A}$. At $\sigma_{B B} / \sigma_{A A}=1.05$, this transition does not occur in the present size range, even though it begins at $N=82$ for LJ clusters. Fig. [4(c) illustrates some of the magic numbers with an antiMackay overlayer that occur for $\sigma_{B B} / \sigma_{A A}=1.05$ and 1.1, all of which have a core-shell geometry.

In summary, we have developed a global optimization approach for binary clusters that is able to locate magic number clusters up to unprecedented sizes, and which should also prove particularly useful for analysing bimetallic clusters. We have applied this approach to binary Lennard-Jones clusters, our hope being that, in the same way as for Lennard-Jones clusters in the one-component case, this system will become a simple archetypal system both to provide candidate structures for a wide variety of binary clusters and to rationalize their structures. Here, we focussed on the case where only the sizes of the two atom types are different. This leads to a remarkable stabilization of polytetrahedral clusters, and a zoo of interesting structures. Because clusters have been found to provide a good indicator of the preferred local structure within supercooled liquids [14, 15], these results can also provide an interesting perspective on the role of size mismatch on glass formation in binary systems. Not only does size mismatch enhance glass formation due to the destabilization of a crystalline solid solution [16], but we see here that it also encourages local icosahedral coordination [17], hence frustrating crystallization further.

J.P.K.D is grateful to the Royal Society for financial support.

[1] D. Sabo, J. D. Doll, and D. L. Freeman, J. Chem. Phys. 121, 847 (2004).

[2] G. Rossi et al., Phys. Rev. Lett. 93, 105503 (2004); A. Rapallo et al., J. Chem. Phys. in press (2005).

[3] J. Jellinek and E. B. Krissinel, Chem. Phys. Lett. 258, 283 (1996).

[4] F. Calvo and E. Yurtsever, Phys. Rev. B 70, 045423 (2004).

[5] D. R. Nelson and F. Spaepen, Solid State Phys. 42, 1 (1989).

[6] S. Cozzini and M. Ronchetti, Phys. Rev. B 53, 12040 (1996).

[7] D. P. Shoemaker and C. B. Shoemaker, in Introduction to Quasicrystals, edited by M. V. Jaric (Academic Press, London, 1988), pp. 1-57.

[8] I. L. Garzón, X. P. Long, R. Kawai, and J. H. Weare, Chem. Phys. Lett. 158, 525 (1989).

[9] A. S. Clarke, R. Kapral, and G. N. Patey, J. Chem. Phys. 101, 2432 (1994).

[10] The Cambridge Cluster Database, http://www-wales.ch.cam.ac.uk/CCD.html

[11] J. A. Northby, J. Chem. Phys. 87, 6166 (1987).

[12] J. P. K. Doye and D. J. Wales, J. Chem. Soc., Faraday Trans. 93, 4233 (1997).

[13] J. P. K. Doye and D. J. Wales, Phys. Rev. Lett. 86, 5719 (2001). 
[14] J. P. K. Doye, D. J. Wales, and S. I. Simdyankin, Faraday Discuss. 118, 159 (2001).

[15] F. C. Frank, Proc. R. Soc. London, Ser. A 215, 43 (1952).

[16] T. Egami and Y. Waseda, J. Non-Cryst. Solids 64, 113
(1984).

[17] H.-J. Lee, T. Cagin, W. L. Johnson, and W. A. Goddard III, J. Chem. Phys. 119, 9858 (2003). 